Agosto 2012 | www.ocula.it - DOI: 10.12977/ocula18

\title{
Il Fiore di Stucco
}

di Stefano Carlucci

Università di Bari-stefanococker@hotmail.com

"Per quanto riguarda il monumento, la sua forma e la decorazione dovrebbe annunciare il suo utilizzo"

Roubo Fils, Traité de la construction des théâtres et des machines théâtrales, Paris 1777, p. 24.

\begin{abstract}
The core of his research paths is theater, considered in its two main components: its physical materialization, the buildings and sites conceived to contain the theatrical event (semiotics of theater architecture) and the dialogue relationship between authors/actors/audience.

These issues originate from the work done in the Teatro Olimpico in Vicenza (1999). Stefano Carlucci also collaborates with the Department of Mechanics, Politecnico di Milano, for the realization of a research project aimed to point out the possible points of contact between forensic engineering and semiotics.
\end{abstract}

\section{Keywords}

Theater Architecture, Reproduction/betrayal, Everyday life/spectacle, Actors/statues

\section{Sommario}

1. Premessa

2. Le prigioni diventano teatro

3. Dallo sfarzo all'oblio

Bibliografia 


\section{Ocula ${ }^{13}$}

Architettura e politica: un incrocio di sguardi | Stefano Carlucci, II fiore di stucco

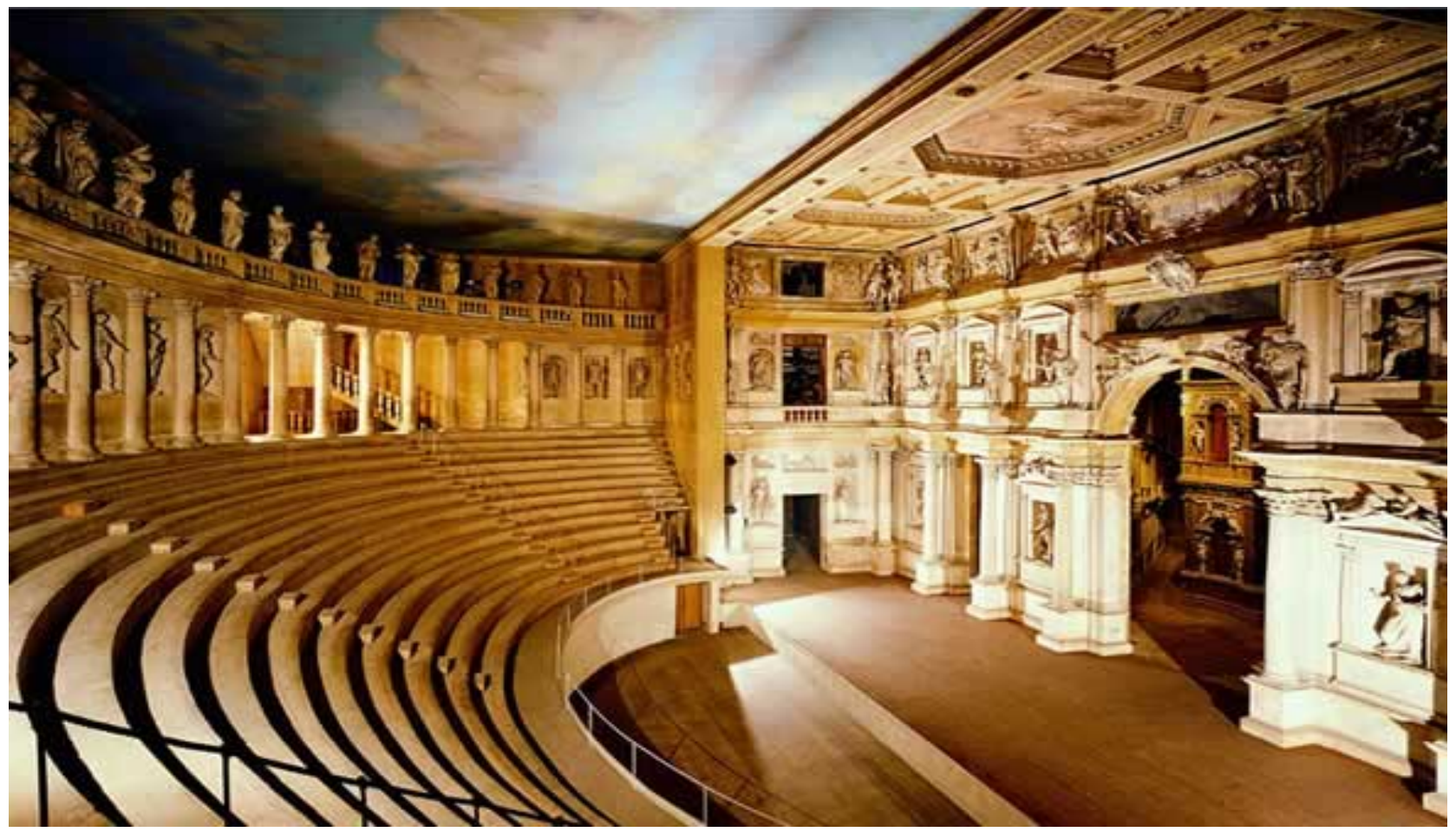

Figura 1.1 Veduta dell'interno del Teatro Olimpico di Vicenza.

"Che cos'è un teatro? Un edificio?.... Il teatro sono gli uomini e le donne che lo fanno. Eppure quando visitiamo i teatri di Drottingholm o di Versailles, il Teatro Farnese di Parma o l'Olimpico di Vicenza, sperimentiamo spesso le stesse reazioni cinestetiche che può darci uno spettacolo vivente. Quelle pietre e quei mattoni diventano spazio vivo anche se non vi si rappresenta nulla. Sono anch'essi un modo di pensare e sognare il teatro, materializzarlo e trasmetterlo per secoli”.

(E. Barba, La canoa di carta, Il Mulino, Bologna 1993, p.153).

\section{Premessa}

Il ruolo rivestito dal Teatro Olimpico di Vicenza nella storia dell'evoluzione del Teatro Occidentale è stato un argomento al centro di numerose analisi e discussioni sin dalla sua costruzione, iniziata nel 1579 e 


\section{Ocula ${ }^{13}$}

Architettura e politica: un incrocio di sguardi | Stefano Carlucci, II fiore di stucco

portata a termine nel 1584, ma per quanto approfonditi e complessi questi studi siano stati, nessuno di essi sembra aver chiarito con certezza l'identità stessa di questo particolarissimo edificio: ultimo dei teatri antichi, primo dei moderni, o cos'altro?

In una moltitudine di definizioni che senza soluzione di continuità arrivano a considerare il Teatro di Vicenza un raffinato esperimento drammatico, piuttosto che un vecchio guscio umido e polveroso, perfetto per accogliere sciami di turisti distratti, l'unica effettiva certezza è la sua sorprendente longevità, dopo più di quattrocento anni difatti sopravvive, fragile ma intatto, come un fiore pietrificato.

Il seguente lavoro si propone quale ulteriore tentativo di spiegare, con un approccio semiotico, il Teatro Olimpico di Vicenza, attore navigato costretto suo malgrado a recitare un doppio ruolo: "ospite forzato" alla mercé di gestori più o meno consapevoli, orgogliosi per il prestigio derivante dal possedere un così decisamente flamboyant salotto buono ma anche infastiditi dagli oneri necessari al suo mantenimento.

Per raggiungere la meta prefissata verranno ricostruite le particolari condizioni, storiche e sociali, che hanno reso possibile l'edificazione del Teatro Olimpico, inquadrandolo in quella breve ma significativa parentesi evolutiva delle maniere e dei luoghi teatrali (Jacquot 1963), che avrebbe portato le rappresentazioni dai mercati e dalle scalinate delle chiese (spazi pubblici), alla cosiddetta "sala all'italiana" (spazi de-privati).

Attraverso una comparazione, effettuata sia con criteri sincronici che diacronici ed imperniata sulla Semiotica dell'Architettura, in particolare di quella teatrale, si traccerà un profilo del Teatro Olimpico, così come suggerito da Marvin Carlson:

A semiotic approach to theatre architecture should encourage us to look not only at the traditional elements of the stage and auditorium but at every distinct element of the theatre complex for what it may reveal about the meanings of this building for this society. Ideally, such analysis should be not only synchronic (considering the relationship between elements at a particular time) but also diachronic (considering temporal changes in elements or in the connotations of elements), since the meanings of those elements that make up a theatre structure, and sometimes the elements themselves, will change as the society that interprets them changes. (Carlson 1987: 9)

È comunque utile sottolineare che qualsiasi evento teatrale, quale che siano le concezioni drammatico-scenografiche che lo hanno originato, si realizzi in uno spazio prestabilito e sia destinato ad essere mostrato ad un pubblico che, nella successiva interpretazione, potrà valutare significanti $\mathrm{e}$ significati in quanto esplicitamente presentati, indirettamente evocati e persino apparentemente ignorati:

Il teatro è effettivamente una pratica che calcola il posto guardato delle cose: se metto lo spettacolo qui, lo spettatore vedrà questo; se lo metto in un altro posto, non lo vedrà e io potrò approfittare di questo nascondiglio per produrre un'illusione: la scena è per l'appunto la linea che taglia il fascio ottico e per così dire il fronte del suo 


\section{Ocula $\mathbf{a}^{13}$}

Architettura e politica: un incrocio di sguardi | Stefano Carlucci, II fiore di stucco

schiudersi: in tal modo si troverebbe fondata, contro la musica (contro il testo) la rappresentazione. (Barthes 1982: 89)

Ma l'eventuale estremizzazione di questa contrapposizione concettuale tra linguaggi scenico-teatrali caratterizzati da percorsi comunicativi prevalentemente simbolico-convenzionali, rispetto ad altri più squisitamente iconico-metaforici o anche metonimici, potrebbe non giovare ad un'analisi di espressioni che pur nella loro innegabile diversità, sono manifestazioni artistiche e che, in quanto tali, raffigurano indirettamente rifuggendo la mera imitazione.

Questo è sicuramente vero anche per il Teatro Olimpico pur con la sua cavea greca, il suo prorompente arco di trionfo (frons scaenae) e la sua minuscola Tebe:

La rappresentazione non si definisce direttamente attraverso l'imitazione: anche sbarazzandosi delle nozioni di "reale", di "verisimile", di "copia”, resterà sempre della"rappresentazione" finché un soggetto (autore, lettore, spettatore od osservatore) dirigerà il suo sguardo verso un orizzonte e vi ritaglierà la base del triangolo di cui il suo occhio (o il suo spirito) sarà il vertice. (Ivi, 97)

In questo studio saranno valutati con attenzione tutti quei processi attraverso i quali il Teatro Olimpico, in quanto artefatto, è da oltre quattro secoli oggetto/soggetto di giudizi e interpretazioni che gli conferiscono significato su vari livelli.

È comunque innegabile che il Teatro Olimpico, come ogni altro edificio costruito primariamente per ospitare eventi drammatici, abbia acquisito negli anni significati secondari (Eco 1968), che nel suo caso particolare sono però probabilmente andati oltre le previsioni dei suoi stessi ideatori.

\section{Le prigioni diventano teatro}

"E insomma i teatri vorriano tutti essere come l'Olimpico di Vicenza, nobilissimo testimonio della splendidezza di quella patria e della magnanimità di quei signori Accademici”.

A. Ingegneri, Della poesia rappresentativa e del modo di rappresentare l favole sceniche, Ferrara, 1598.

Le parole di Angelo Ingegneri, incaricato di curare regia e luci per la Prima dell'Edipo Tiranno, versione in endecasillabi sciolti dell'Edipo Re di Sofocle, sarebbero risultate prive di senso se pronunciate solo qualche anno prima del 1585 , anno in cui fu inaugurato il teatro. 


\section{Ocula ${ }^{13}$}

Architettura e politica: un incrocio di sguardi | Stefano Carlucci, II fiore di stucco

L'evento che suscitò tanto interesse nelle principali corti europee ${ }^{1}$ dell'epoca per la bellezza del complesso teatrale e la magnificenza di scene e costumi, si era difatti svolto in un vecchio castello fluviale di origine medioevale, divenuto nel corso dei secoli posto di dogana, monastero e prigione.

Il celebre architetto Andrea Palladio viene incaricato nel 1579 dall'Accademia Olimpica di Vicenza di progettare un teatro "alla maniera degli antichi" all'interno di un vecchio castello situato sulle rive del Bacchiglione, il fiume che attraversa Vicenza.

In disarmonia rispetto alle sue abitudini costruttive Palladio non può però in questo caso fornire a quella che è destinata a rimanere la sua ultima creatura di un significante architettonicamente (una facciata) che ne testimoni pubblicamente l'identità e le funzioni drammatiche.

Ispirandosi alle teorie filosofiche di ispirazione neoplatonica molte diffuse nell'Italia rinascimentale, Palladio era solito dotare le sue opere di una "organicità strutturale", grazie alla quale interno ed esterno dell'edificio, pubblico o privato che fosse, risultassero indissolubilmente collegati da un rapporto di dialogica permeabilità, entrambi le componenti dovevano in qualche modo riflettere un principio di armonia universale:

Ho dotato di frontespizio le facciate principali di tutte le ville ad anche di qualche municipio...poiché queste facciate monumentali mostrano l'ingresso della casa e aggiungono molto allo splendore e alla magnificenza dell'opera, la facciata dunque deve essere fatta più importante del resto. (Palladio 1570: 5)

Il Teatro Olimpico si connota quindi sin dall'inizio come un'irripetibile eccezione nella ampia attività architettonica dell'architetto veneto, si pensi ad esempio alla lunga e fortunata collaborazione instaurata con Paolo Veronese, la cui testimonianza più significativa è probabilmente la Villa Barbaro-Volpi in provincia di Treviso.

Gli affreschi che adornano questa villa situata a Maser rappresentano il perfetto connubio tra spazio architettonico e spazio pittorico, unione in virtù della quale le sale interne riescono in qualche modo ad echeggiare il senso di spazialità ariosa della campagna esterna.

Non soffermandosi ora sulle motivazioni che non hanno permesso al teatro di avere un volto esterno, che possono comunque essere in linea generica addebitate alla limitatezza dei mezzi dei suoi stessi committenti, risulta interessante sottolineare che persino oggi un qualsiasi visitatore che non conoscesse la storia del teatro o la sua stessa esistenza, avrebbe serie difficoltà ad individuarlo dall'esterno.

Se è vero che l'abito non fa il monaco è innegabile che in qualche modo un vestito rappresenti chi lo indossa, mettendolo in comunicazione con il mondo esterno e contribuendo a generare catene di interpretanti che a volte,

1 «At carnival time, March 3,1585, the new theatre was opened with a magnificent performance of Aedipus the King. Present not only the nobility from Venice but the Empress Maria of Austria and the ambassador of France.» (G. R. Kernodle 1944: 169).

5 | www.ocula.it | agosto 2012 


\section{Ocula ${ }^{13}$}

Architettura e politica: un incrocio di sguardi | Stefano Carlucci, II fiore di stucco

il caso qui esaminato ne è un esempio lampante, possono condurre a conclusioni più o meno giuste.

In effetti ciò che si vede ancora oggi percorrendo il cortile interno del castello (solido e funzionale) non lascia in alcun modo intravedere la prodigalità architettonica del teatro (fragile ed eccedente), la sua identità drammatica e le sobrie e robuste mura che lo racchiudono si mimetizzano perfettamente con quella parte del centro di Vicenza che conserva la sua identità commerciale lontana dalla elegante euritmia (armonia delle parti che compongono un oggetto) che Palladio aveva individuato quale collante ispiratore delle sue realizzazioni.

Quello stesso centro urbano in cui Palladio aveva concentrato negli anni precedenti una buona parte delle sue opere più famose: la Loggia del Capitanio, La Basilica della Ragione, Palazzo Chiericati.

In questo contesto storico-sociale un gruppo di umanisti riunitisi in Accademia $^{2}$, affascinati dai fasti della civiltà classica e desiderosi di affrancarsi, almeno simbolicamente, dall'odiato controllo veneziano, decidono, dopo aver organizzato per svariati anni una serie di allestimenti effimeri in occasione di particolari ricorrenze3, di costruire un teatro stabile, scrigno ideale per i loro esercizi drammatici ma anche testimonianza duratura del loro potere politico e culturale.

È utile ricordare che la valenza di un simbolo è collegata alla sua comprensibilità all'interno di un sistema comunicativo condiviso, al di fuori del quale la sua significatività risulta mutevolmente diminuita.

In questo caso particolare l'ambizioso piano degli Accademici risulta quindi fatalmente destinato al fallimento sin dalla sua ideazione: la desiderata dimostrazione di prestigio di quella che si considerava in maniera autoreferenziale una classe dirigente colta e raffinata, sarebbe rimasta un'opera incompiuta e urbanisticamente poco rilevante.

Il sogno classicheggiante dell'èlite vicentina si connota difatti come un percorso interpretativo implicito (cfr., Ponzio 2004: 34) per la gran parte della città, per essere esplicitato solo per pochi eletti in particolari occasioni.

In definitiva il Teatro Olimpico può essere definito come una reinterpretazione (consapevolmente?) libera ed "infedele" di un teatro antico, racchiusa suo malgrado dentro un guscio tutt'altro che armonioso, almeno secondo i canoni generalmente seguiti dallo stesso Palladio:

Although the desire to construct slavish imitations of Classical theatres was clear, the primary obstacle to this dream of illusory restoration was economical. For no prince, town, or Academy had the means to construct those immense stone theatres whose imposing ruins still dominated the surrounding buildings. (Schiavo 1987: 95)

2 L’Accademia Olimpica viene fondata a Vicenza nel 1555 e si distingue da altre organizzazioni similari dello stesso periodo, per il fatto di non essere riservata solo a nobili di nascita e di accogliere fra i suoi membri persone dalla provenienza più diversa. Lo stesso Andrea Palladio (Andrea della Gondola) era figlio di uno scalpellino padovano.

3 Circa le iniziative più rilevanti dell'Accademia Olimpica si ricordano: la rappresentazione pubblica dell'Andria di Terenzio nel 1556, l'organizzazione dei "Giochi Olimpici" in onore di Ercole, patrono dell'Accademia stessa, la messa in scena della Sofonisba di Giangiorgio Trissino nel 1576.

6 | www.ocula.it | agosto 2012 


\section{Ocula ${ }^{13}$}

Architettura e politica: un incrocio di sguardi | Stefano Carlucci, II fiore di stucco

L'Olimpico nasce dunque ispirato da ideali di splendore ed armonia fondati sul modello classico, ma è irrimediabilmente condizionato dalla situazione effettiva di una città politicamente ed economicamente subalterna, destino al quale non può in alcun modo sottrarsi.

Ottenuti quindi dal comune di Vicenza in concessione una parte del suddetto castello doganale (delibera comunale del 24 febbraio 1580) i lavori possono partire, avendo come vincolo iniziale quello di non dover intaccare in alcun modo la struttura muraria portante, paletti progettuali a cui in seguito si aggiungeranno molte altre limitazioni. 4

$\mathrm{Si}$ attua così una procedura che per certi versi ricorda la fecondazione artificiale eterologa con ovodonazione, anche qui infatti l'ovulo/significante viene svuotato per ospitare un nucleo/significato completamente nuovo. La significanza cangiante del castello si arricchisce così di percorsi interpretativi nuovi, anche se non manifesti, in cui esterno ed interno devono, nonostante le inevitabili difficoltà, comunque convivere.

Le strutture murarie mantengono così una funzione protettivo-celante paragonabile a quella che riveste il bozzolo per la crisalide, con la non secondaria differenza però che in questo caso particolare la simbiosi architettonica, diversamente da altre di tipo biologico, è concepita per durare e non finalizzata alla rottura del guscio, senza il quale questo complesso architettonico geneticamente modificato non potrebbe esistere.

Proprio in conseguenza dell'esiguo spazio a disposizione il progetto palladiano di edificare un teatro sul modello di quelli greci e romani sarebbe risultato di realizzazione quantomeno problematica.

A questa difficoltà materiale l'architetto cercò di porre in qualche modo rimedio anche grazie ad una rinnovata creatività.

Una evoluzione metodologica di questo tipo è testimoniata anche da James Ackermann, il quale distingue due fasi principali nell'approccio teorico all'architettura di Palladio: nella prima i modelli antichi vengono accuratamente studiati, per essere poi, con le eventuali modifiche, riproposti; nella seconda, in cui le lezioni del passato sono state abbondantemente metabolizzate, l'ormai maturo architetto, si sente libero di poter "trasgredire" i rigidi principi classici per adattarli alle esigenze moderne.

A Palladio sono comunque ben chiare le indicazioni sulle proporzioni architettoniche che l'edificio teatrale deve seguire secondo i dettami di Vitruvio, esposti nel famoso e controverso passaggio tratto dalla riedizione dei Dieci libri di Architettura, curata da Daniele Barbaro nel 1556, volume di cui lo stesso Palladio disegna le illustrazioni:

Il piano del teatro stesso deve essere costruito come segue. Dopo aver fissato il centro principale tracciamo una linea di circonferenza equivalente a quello che è il

4 «The Accademia could not remove the timbers, iron hardware, or other material which had been used for the maintenance and fortification of the site, and particularly not the roofed portion...the land granted to the Accademia was only large enough to house the auditorium, stage, and proscenio, but lacked sufficient space for the perspectives which Palladio proposed.» (H. G. Myers, 1978: 22).

7 | www.ocula.it | agosto 2012 


\section{Ocula ${ }^{13}$}

Architettura e politica: un incrocio di sguardi | Stefano Carlucci, II fiore di stucco

perimetro basso, e quindi all'interno di essa iscriviamo quattro triangoli equivalenti a distanze uguali tali che tocchino la linea di confine del cerchio...considerando di questi triangoli quello il cui lato è più vicino alla scena, lasciamo che la parte anteriore della scena sia determinata dalla linea data da quel lato che taglia un segmento del cerchio, e disegnamo, attraverso il centro, una linea parallela a partire da tale posizione, in modo da separare la piattaforma del palco dallo spazio dell'orchestra. (Vitruvius 1960: 146)

Partendo dalla sua personale interpretazione di tali regole Palladio riesce ad adattarle sapientemente in funzione delle suddette limitazioni spaziali, e nell'Olimpico la cavea si deforma sino a diventare una porzione di ellisse, distaccandosi marcatamente dalla regola vitruviana, la quale prescrive invece un arco di cerchio il cui raggio dovrebbe equivalere ad una volta e mezzo il diametro dell'orchestra.

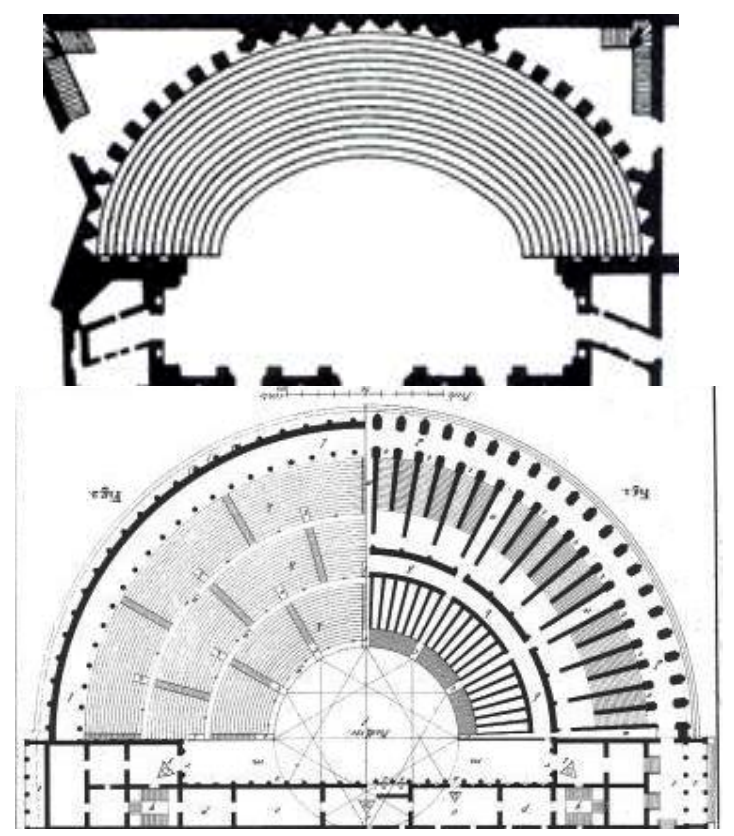

Figura 2.1 Pianta del Teatro Olimpico.

Figura 2.2 Pianta del Teatro Romano secondo Vitruvio.

L'effetto così ottenuto, grazie anche ad una ricercata alternanza tra pieni e vuoti che si estende dal loggione superiore sino alla frons scaenae, fa sì che lo spazio percepito sembri ampliarsi fino quasi a dissimulare gli angusti limiti spaziali.

Se e in che misura il Teatro Olimpico sia effettivamente fedele al progetto palladiano originale è un punto che ha causato svariate analisi e generato discordanti conclusioni, ma ciò che sembra comunque condivisibile è che il successivo intervento di Vincenzo Scamozzi5, necessario per la sopraggiunta

5 «Nel maggio del 1584 l'Accademia prende contatto con Vincenzo Scamozzi e gli affida il progetto delle prospettive, quindi la realizzazione delle strade di una Tebe immaginaria. Questi si ispira alla scena semipraticabile teorizzata da Peruzzi e dal Serlio.» (Beyer, op. cit.: 40). 


\section{Ocula ${ }^{13}$}

Architettura e politica: un incrocio di sguardi | Stefano Carlucci, II fiore di stucco

morte di Palladio e il conseguente affidamento temporaneo del cantiere al figlio di Palladio Silla, abbia comunque alterato gli equilibri originari, dando un risalto superiore alle scenografie, molto di più di quanto lo stesso Palladio aveva con ogni probabilità immaginato.

A questo proposito alcuni critici tendono a sottolineare una sorta di "rottura" nell'interazione architettonico-drammaturgica degli elementi costitutivi dell'intero edificio, causata da un contrasto tra le scene ed il resto del teatro:

Scamozzi allargò le porte della frons scaenae per intensificare l'illusione spaziale a scapito del principio di proporzionalità, di quell'armoniosa corrispondenza delle diverse parti fra loro e con il tutto, che era stato rigidamente seguito da Palladio. Quindi la tribuna degli spettatori, il proscenio e la frons scaenae non mantenevano più fra di loro un rapporto di equilibrio. (Beyer, op. cit.: 42)

A testimonianza dei rapporti non proprio buoni fra i due celebri architetti e della diversità di vedute su alcune tematiche fondamentali, si può citare la testimonianza di un loro omologo inglese, Inigo Jones, che descrive l'atteggiamento di Scamozzi, personalmente conosciuto nel 1613 durante una sua breve visita in Italia, come fortemente negativo nei confronti del suo più anziano collega, verso il quale non manca di manifestare apertamente "malignità e pregiudizi" (Zorzi 1965: 302).

Riguardo il progetto originale di Palladio, in particolare per l'apparato scenico, è ragionevole pensare che egli avesse comunque previsto un qualche tipo di apparato "duttile", probabilmente una reinterpretazione in chiave moderna degli antichi peryaktoi, quei prismi girevoli che nel teatro greco erano posti ai lati del proscenio e servivano ad accompagnare visivamente i cambiamenti di luogo.

Grazie a questo accorgimento, dalla non difficile realizzazione, si sarebbe ottenuta una notevole poliedricità performativa che avrebbe permesso al teatro di passare facilmente dalla tragedia classica ai generi più diversi:

They may have been designed originally to be either permanent or temporary. Since the deliberations speak at one point of performing both a Pastorale and a Tragedy in the same theatre, they may have been a generalized types of perspective which could be used for all types of theatrical performances. (Myers: 138)

Gli eventi succedutisi in seguito hanno vanificato queste potenzialità drammatiche in un edificio teatrale che invece conserva, sin dal 1585 , le prospettive lignee che riproducono tridimensionalmente una città immaginaria a metà tra una mitologica Tebe, con le sue famose sette vie confluenti in un unico punto, ed una sognata Vicenza, materializzazione classicheggiante di un ideale. 


\section{Ocula ${ }^{13}$}

Architettura e politica: un incrocio di sguardi | Stefano Carlucci, II fiore di stucco

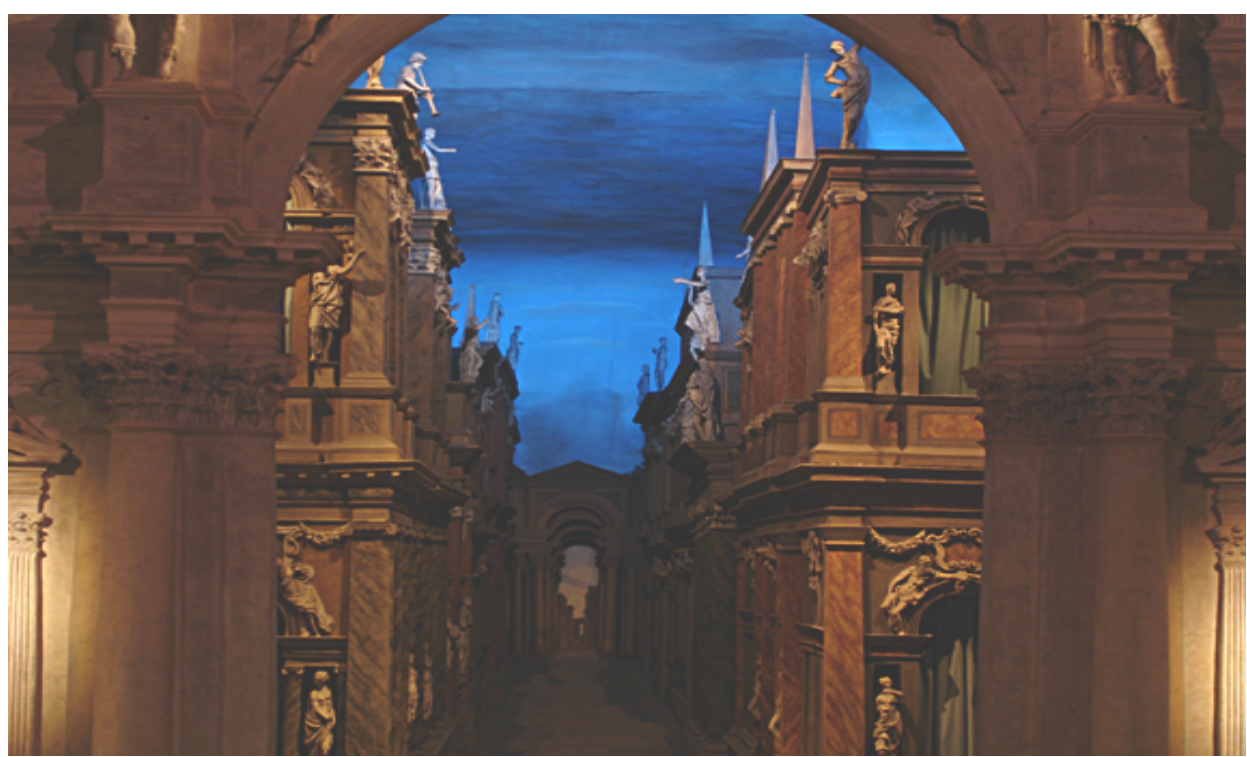

Figura 2.3 Teatro Olimpico, veduta Ianua Regia e scorcio prospettive interne.

Il teatro trova così il suo kunstwollen (volere/destino artistico), croce $\mathrm{e}$ delizia di un edificio destinato ad impersonare per sempre un sogno bellissimo ma tragicamente immobile.

Ed è proprio in conseguenza di questa sua immutabile fissità che l'Olimpico, rincorrendo i fasti e le illusioni di un'epoca lontana, si astrae dalla quotidianità per esistere fuori da essa, in un tempo altro, perfetta sublimazione di un attimo storico irripetibile e quindi già anacronistico appena qualche anno dopo la sua costruzione:

Palladio accetta e fa propria la scelta, ormai anacronistica, della restituzione del teatro vitruviano, trascurando di proposito i risultati conseguiti dalla più evoluta tecnica teatrale contemporanea; inoltre forza spregiudicatamente fino all'iperbole i termini stessi di quella scelta, valutando appieno le ragioni profonde che l'avevano motivata. Senza ombra di dubbio l'organismo che ne risulta rivela, specie ne proscenio, il suo significato di retorica parata glorificante di una classe e di un potere. (Molinari 1974: 318)

Ulteriore prova di questa sua unicità è il fatto che, nonostante i numerosi attestati di ammirazione, il Teatro Olimpico sia rimasto fino ad oggi un modello di architettura teatrale pressoché inimitato.

Lo stesso Vincenzo Scamozzi che si occuperà qualche anno dopo della realizzazione ex novo del Teatro di Sabbioneta, la città simbolo del potere dei Gonzaga in Italia, conformerà la sua nuova creatura ad esigenze politicorappresentative molto diverse.

Nel teatro di Sabbioneta si assiste al passaggio da un'organizzazione dello spazio interno che rispecchia l'oligarchica ripartizione del potere distintivo dell'Accademia Olimpica (sette strade= suddivisione paritaria del potere fra $\mathrm{i}$ suoi membri, spesso definiti "a club of equals"), ad uno spazio scenico che ha come punto focale unico ed onnicomprensivo una sorta di "royal box" ante 


\section{Ocula ${ }^{13}$}

Architettura e politica: un incrocio di sguardi | Stefano Carlucci, II fiore di stucco

litteram posta al centro di una cavea che fra l'altro si distacca completamente, per dimensioni e forma, dalle prescrizioni concepite da Vitruvio.

Da questa posizione privilegiata il Signore del teatro di Sabbioneta ha il controllo totale su tutto quello che accade sul palco e fra il pubblico, dando vita così, all'interno di uno spazio fisico/drammatico ristretto, a quell'ideale di assolutismo politico che nei secoli seguenti si allargherà materialmente ai confini dei moderni stati nazionali europei.

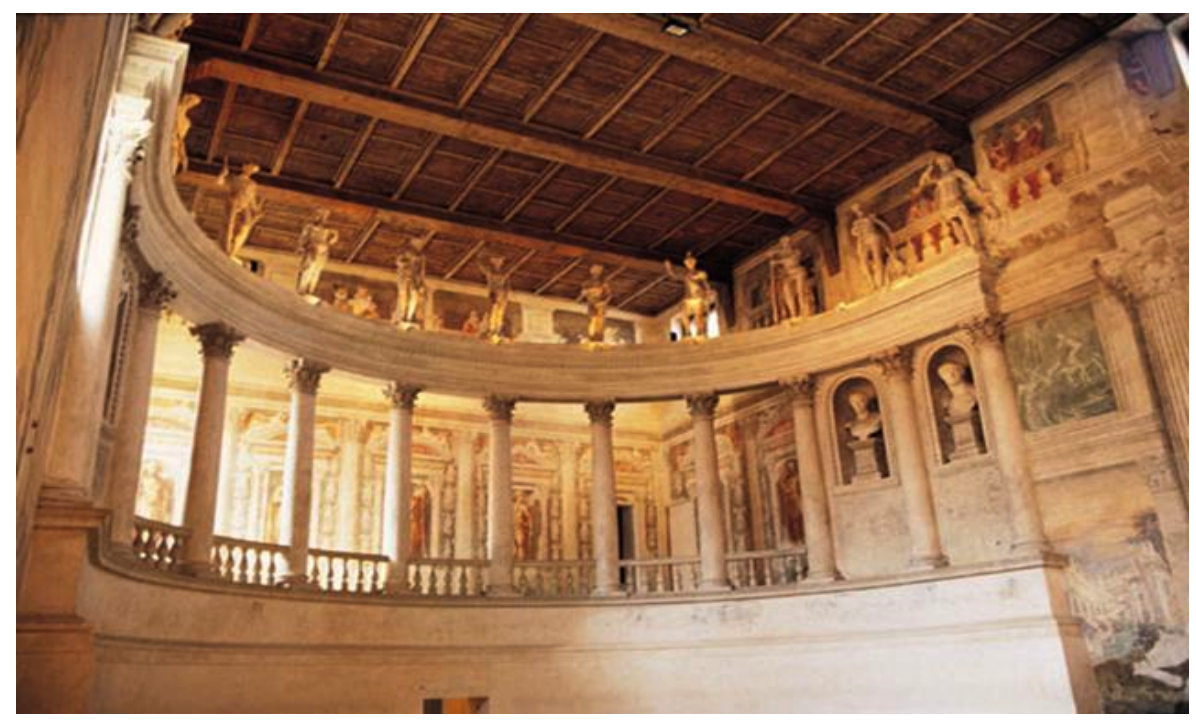

Figura 2.4 Teatro di Sabbioneta.

Fra i giudizi che interessano il Teatro Olimpico di Vicenza è possibile individuare due macro-categorie: nella prima si assiste ad un riconoscimento pressoché unanime della sua universalmente riconosciuta bellezza, nell'altra si fa strada l'opinione secondo la quale il teatro sia in qualche modo ineptus, non adatto, in definitiva niente di più di un prezioso quanto scomodo giocattolo drammatico.

È difatti evidente che nel rapporto tra immaginazione e visibilità il Teatro Olimpico di Vicenza persegua una resa del luogo dell'azione drammatica improntata alla ricostruzione materiale della Tebe sofoclea luogo dell'azione dell'Edipo Tiranno.

Il proscenio interpreta l'ideale rinascimentale di piazza centrale quale punto d'incontro delle strade della città, un miraggio rinascimentale ricorrente, associato a principi di ordine e decoro, spesso opposti alle contingenze della realtà quotidiana, come nel caso della Vicenza cinquecentesca sconvolta dalla peste, urbanisticamente ancora molto legata ad un'identità medioevale e, dal punto di vista politico, oramai quasi rassegnata all'ingombrante giogo veneziano.

Per quanto concerne il Teatro Olimpico, però, lo scarto interpretativo possibile è limitato da questa ricercata somiglianza coincidente tra luogo 


\section{Ocula $\mathbf{a}^{13}$}

Architettura e politica: un incrocio di sguardi | Stefano Carlucci, II fiore di stucco

dell'azione e scene, se è vero che lo stesso Ingegneri, corago ${ }^{6}$ della sfarzosa prima del 1585, riconosce nella traboccante personalità architettonica delle stesse un limite ad un utilizzo diversificato del teatro:

Egli è il vero che quello è un apparato più tragico che comico e in niuna guisa pastorale; tuttavia con mutazioni e aggiunte a proposito potrebbe tornar bene a tutte le cose. Ma per le tragedie io vi scorgo un convenevolenzagrandissima che quella fronte, la qualesecondo l'uso degli antichi, non vuole figurare altro che un qualche illustre edificio fatto per ornamento di quella città che si piglia a rappresentare. (Ingegneri 1598: 26)

Le sue ricercatissime stradine e il suo ornato frons scaenae, sono difatti perfetti per ricreare l'antica Tebe, ma anche difficilmente conciliabili con qualsiasi altro tipo di rappresentazione diversa dalla tragedia antica:

...although Palladio's building with his much frons scaenae was much admired, it was too impractical a building to be copied. It is not, as usually said, the first modern theatre but one of the last and the greatest of the Renaissance academic theatres. (Hartnoll, 1967: 36)

\section{Dallo Sfarzo all'Oblio}

The hour of lowering the courtain having arrived, first there was a sweet odor of perfumes... Then, in a twinkle of the eye, the taut curtain fell before the stage. Here it would be difficult to express in words, or even to imagine, the great joy and immensurable pleasure which came upon the spectators at the sight (F. Pigafetta, lettera del 1585, pubblicata nella Raccolta Milanese, 1756-57, copia presente nella Yale-Rockfeller Theatre Collection, tradotta in inlgese da G. R. Kernodle).

Nella descrizione ammirata di Filippo Pigafetta sono sintetizzate alcune delle probabili sensazioni che i fortunati spettatori dell'Edipo Tiranno del 1585 dovettero provare nell'assistere ad un evento così lungamente preparato. Dopo anni di allestimenti effimeri e a poco più di un lustro dall'inizio dei lavori, la creatura teatrale così fortemente voluta dai membri dell'Accademia Olimpica è finalmente pronta ad accogliere i suoi prestigiosi ospiti.

Per onorare al meglio questo evento viene scelto l'Edipo Tiranno di Orsetto Giustiniani, adattamento moderno dell'Edipo Re di Sofocle, che viene preferito ad una pastorale scritta da Fabio Pace, scelta iniziale.

Si tratta indubbiamente di un cambiamento di non poco conto che ha sicuramente finito per influire sulle stesse scenografie le quali, in armonia

${ }^{6}$ Figura corrispondente al regista nel teatro moderno.

12 | www.ocula.it | agosto 2012 
con la scelta iniziale della pastorale, avrebbero dovuto raffigurare un paesaggio silvestre e non le sette vie di Tebe.

L'Olimpico sceglie quindi per la sua inaugurazione il genere alto, la tragedia classica, e l'ideale eroico perseguito dai suoi creatori rivive dopo molti secoli nei versi dell'opera sofoclea riadattata da Orsatto Giustiniani.

Lo stesso Pigafetta continua nella sua entusiastica descrizione parlando di circa tremila persone presenti all'evento, dato però a cui difficilmente si può prestare fede, soprattutto in considerazione delle dimensioni interne del teatro, che doveva comunque essere colmo all'inverosimile, come testimoniato da altre fonti, non tutte in verità così benevoli.

Antonio Riccoboni, un altro dei presenti, non manca difatti di evidenziare l'evidente contrasto fra l'esibizione di sfarzo a cui sta assistendo e la situazione, a dir poco critica, che c'è in quel momento a Vicenza:

Mi parve strano che in un tempo calamitosissimo di peste si adoperassero quelli vesti tanto pompose... E se bene alcuni dicono che ciò si può fare per dare maggiore speranza al popolo, mi pare che si deve dare speranza con altro che con le vesti e che in tempo tanto misero et calamitoso non si doveva andare nello estremo di pompa così solenne...Ma concediamo loro questo modo pomposo di vestire, corrispondente forse maggiormente alla magnificenza de Signori Accademici che a un tempo di peste. (Riccoboni, 1973: 39-51)

In una sorta di riformulazione dell'odi profanum vulgus et arceo oraziano, si verifica qui la folgorante esplosione di un universo scintillante e artificioso completamente alienato dalla realtà che lo circonda: una notevole quantità di denaro, accumulato attraverso un'autotassazione forzosa operata dai suoi stessi costruttori, è servita ad erigere un piccolo gioiello drammatico, fragile però al punto di non potersi rapportare direttamente con la realtà, dalla quale invece si isola, proprio come gli stessi accademici, incapaci di amministrare la loro stessa città.

Lo sfarzo mostrato in questa rappresentazione stride marcatamente con la situazione di una comunità che ha da poco superato una gravissima epidemia di peste, di cui porta ancora i segni, e che malgrado tutto esiste politicamente sotto l'ingombrante ombra della Serenissima Repubblica di Venezia.

Ma è forse proprio in conseguenza di questo rapporto quasi inesistente con il reale, la mancanza di un sostrato socialmente rilevante su cui attecchire, che questo avvenimento così celebrato rimarrà unico. Non è sicuramente un caso che, pur avendo l'Olimpico a disposizione, già all'inizio del secolo successivo a Vicenza vengano edificati nuovi edifici teatrali, il Teatro delle Garzerie ad esempio, più adatti alle nuove esigenze ed ai nuovi gusti drammatici: ai costosi sogni di pochi si preferiscono le necessità concrete di tanti.

Riguardo la complessa organizzazione della prima dell'Olimpico, Ingegneri dispone che ci siano solo nove attori recitanti, circa ottanta comparse e che il coro sia composto da quindici elementi.

Il pubblico, comunque molto numeroso, è di estrazione sociale relativamente mista, anche se come era abitudine diffusa a quei tempi, 
orchestra e cavea sono riservate alle personalità più importanti e uomini e donne sono rigidamente separati, avendo queste ultime assegnate le prime tre file della cavea, per godere di una migliore visione.

Cavea che, in quanto tale, subirà nel corso del tempo notevoli modifiche fino a scomparire progressivamente: già nel suddetto teatro di Sabbioneta lo spazio riservato al pubblico sarà molto lontano dall'ergonomica semplicità della cavea palladiana e si connoterà come testimonianza di un processo evolutivo secondo il quale a poco a poco le "democratiche scalinate" lasceranno il posto ai più settari palchi.

Lo spettacolo in un certo senso finirà gradatamente per spostarsi dal palco ai settori riservati al pubblico, più attento a cercare di conquistare un buon posto, che ad avere un'estesa visibilità della rappresentazione stessa e i teatri con uno spazio destinato al pubblico organizzato come quello dell'Olimpico diventeranno poco a poco:

...too contrary to our customs and manners. We have becomed accustomed to boxes; they allow anyone to attend the theatre according to his rank and means and to gather there with his usual social companion. (Patte, 1782: 1965)

Nel caso dell'Olimpico invece la maggiore soddisfazione possibile rimane probabilmente quella di fare parte di quel ristretto cerchio composto da novantacinque "spettatori imperituri", quelle statue che non hanno bisogno di vanagloriosi palchi e che dal 1585 non si sono persi un singolo evento. 7

In un contesto sociale in via di cambiamento si confrontano dei modi di intendere il teatro per certi versi opposti: accademico ed esclusivo/escludente quello dell'Olimpico, più moderno e socialmente rappresentativo/accogliente quello dei teatri ad esso successivi.

Da una separazione netta tra espressioni teatrali accademiche e popolari caratteristica della fine del Cinquecento in Italia, si arriverà gradualmente ad una sorta di commistione fra i diversi tipi di pubblico, che verranno materialmente riuniti nei teatri Barocchi, rimanendo però rigidamente separati nei palchi.

\footnotetext{
7 In realtà non è proprio così: le statue che adornano la scaena frons sono ad essa contemporanee mentre quelle che sormontano il loggione furono aggiunte nel Diciottesimo secolo. Risulta curioso evidenziare come in origine le statue dovessero personificare divinità e personaggi mitici ed imperatori romani, in ossequio alle tradizioni a cui l'Accademia Olimpica si ricollegava idealmente. In seguito però, per giustificare ulteriori esborsi necessari al compimento dei lavori, i munifici committenti furono ricompensati con la sublimazione più prestigiosa, entrando di diritto nel nuovo Pantheon di legno e stucco. Ci furono però delle conseguenze per questo imprevisto cambiamento ed al giorno d'oggi è possibile notare alcune statue che presentano caratteri fisici marcatamente femminili (seni) accoppiati volti maschili.
} 


\section{Ocula ${ }^{13}$}

Architettura e politica: un incrocio di sguardi | Stefano Carlucci, II fiore di stucco

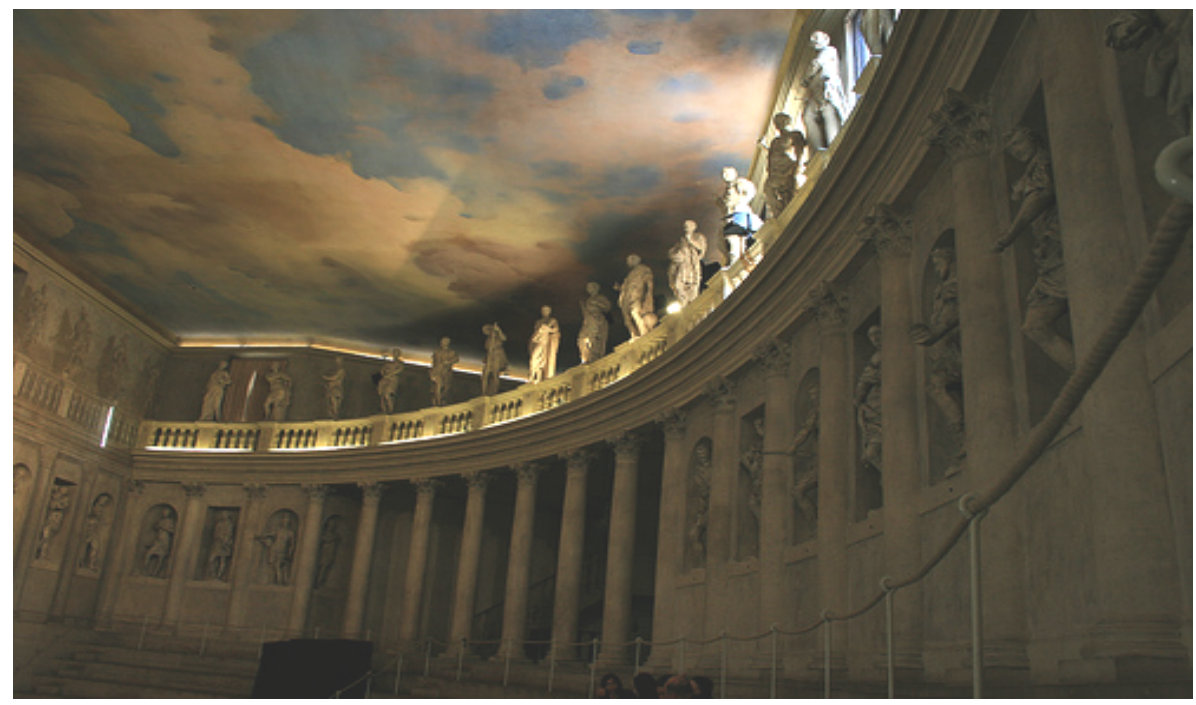

Figura 3.1 Teatro Olimpico, colonnato sovrastante la cavea e statue.

Il teatro come cartina tornasole o traccia dei cambiamenti sociali all'interno di una comunità: l'ideale classicheggiante degli Accademici Olimpici rimarrà però solo un embrione, differenziandosi per questo motivo sia rispetto ai modelli antichi a cui si ispirava ${ }^{8}$, che agli edifici teatrali che gli sarebbero succeduti nel corso della storia del teatro Occidentale.

The cultural view of the theatre as s sort of public monument was totally absent in the medieval and Renaissance concepts of theatrical space. It was not until the baroque period that something similar to the classical view appeared as in the placement of theatres within a urban design. (Carlson, op. cit.: 68)

I teatri costruiti a partire dal Diciassettesimo secolo in tutta Europa tenderanno difatti ad essere sempre più inseriti nel contesto sociale e ad acquistare una crescente visibilità all'interno dello stesso, l'Olimpico rimane forse uno degli esempi più significativi della "asocialità" di un certo tipo di teatro.

In fin dei conti pur essendo collocato in una posizione urbanisticamente centrale, il che lo differenzia ad esempio dai contemporanei teatri pubblici Inglesi, il Teatro Olimpico è fin dalla sua costruzione una sorta di "corpo estraneo" rispetto alla complessità socioculturale della città che lo ospita.

The court theatres of the Renaissance and their architectural successors...were small, ornate, secluded halls, removed not only from external nature but from the view, indeed even from the consciousness of all but those selected few who were permitted to enter them. Nothing could be more unlike such performance spaces than the huge open theatres of classical times. (Ivi: 61-62)

8 Il teatro classico, Greco e Romano, era nelle sue diverse declinazioni un teatro "aperto": si pensi alle immense scalinate di cui erano composte le cavee dei teatri greci e al fatto che nelle città della Grecia Classica gli eventi teatrali pur non essendo del tutto gratuiti erano frequentati da buona parte della popolazione grazie anche a sovvenzioni ed incentivi pubblici. 


\section{Ocula ${ }^{13}$}

Architettura e politica: un incrocio di sguardi | Stefano Carlucci, II fiore di stucco

Le stesse "arene di galli" londinesi (Shakespeare, Henry V, Atto I, Prologo, vv. 12), pur essendo collocate oltre i confini della city, fuori quindi dal controllo delle autorità cittadine, maldisposte nei confronti del teatro per le loro convinzioni religiose di stampo Puritano, riuscivano ad attirare un numero di spettatori tutt'altro che trascurabile: circa il 10\% della Londra a cavallo tra il Sedicesimo ed il Diciassettesimo Secolo andava settimanalmente a teatro, nonostante le suddette avversità.

I teatri pubblici inglesi di fine Cinquecento erano aperti a tutte le classi sociali a differenza dell'Olimpico, isolato da quella vita quotidiana che invece permeava nel profondo il teatro di Shakespeare:

And by their very size, their capacity for holding large numbers of people, the English theatres were public theatres in the classic sense, more truly vitruvian in spirit than any courtly or academic adaptation, with its limited audience. (Yates 1969: 129)

Paradossalmente gli edifici teatrali italiani simili all'Olimpico in qualche misura tradivano i modelli antichi a cui si ispiravano, dando vita a delle traduzioni a volte discutibili per fedeltà ed accuratezza:

The italian princes of the Renaissance, seeking to revive in their own domains the departed glories of classical civilization began the staging of classical drama in theatrical spaces based on what were thought to be classical architecture principles... Vitruvius De Architectura was rediscovered in 1414. Even had the interpretation of these sources totally accurate, however the altered social structure of Renaissance Italy placed theatre in a very different social context from that of ancient times, and the physical configuration of the new theatres not surprisingly reflected this. (Carlson, op. cit.: 38)

I teatri pubblici Elisabettiani erano tradizionalmente più moderni rispetto all'Olimpico, essendo concepiti per portare dei ricavi economici ai suoi proprietari nonché agli stessi attori, dimostrando anche per questo aspetto di essere una parte attiva dell'organismo urbano e non un "illustre sconosciuto".

Il Teatro Olimpico si connota invece quasi fosse un allestimento effimero, si pensi agli ingressi trionfali del Quattrocento e del Cinquecento o alle di poco successive Masks inglesi, che ha smentito probabilmente i progetti dei suoi stessi costruttori, sopravvivendo alla caducità dei materiali da cui è composto (legno di abete per la cavea, mattoni e stucco per il resto).

In definitiva il Teatro Olimpico non sarebbe mai diventato "a place of seeing in a double sense" (Barthes 1982) così come lo erano stati i suoi illustri progenitori, quali ad esempio il Teatro di Taormina, quello di Pergamo o quello di Efeso.

Ulteriore elemento fondamentale per la riuscita della rappresentazione del 3 marzo del 1585 è il sapiente utilizzo delle luci: la natura coperta della struttura, unita alla continua alternanza di volumi, richiede una complessa studio per far in modo che eventuali illuminazioni non provochino luci ed 


\section{Ocula ${ }^{13}$}

Architettura e politica: un incrocio di sguardi | Stefano Carlucci, II fiore di stucco

ombre indesiderate. Per ovviare a questo problema Angelo Ingegneri e Vincenzo Scamozzi si dividono i compiti: il primo si occupa quindi dell'illuminazione della sala, il secondo delle luci interne alle scene, ottenendo peraltro un ottimo risultato, come dichiara in una lettera dell'epoca Giacomo Dolfin', missiva in cui esalta la bellezza delle prospettive illuminate alla perfezione.

Dopo oltre quattro ore, alle cinque del mattino, cala il sipario su questo evento e una breve ma significativa stagione del teatro si chiude simbolicamente: nel volgere di pochi anni l'interesse per la tragedia classica andrà difatti scemando, soppiantato dal fascino e dalla incontrastabile diffusione di nuove forme teatrali, tutte indifferentemente "a disagio" all'interno del guscio palladiano.

Stando così le cose il Teatro Olimpico, proprio per la sua ingombrante personalità architettonico-performativa, deve quindi rassegnarsi ad lungo oblio, dal quale è episodicamente ridestato, avendo sempre però l'assoluta consapevolezza di non poter in alcun modo ripetere la magia di quella notte di marzo del 1585:

Dopo la trionfale rappresentazione dell'Edipo Tiranno un silenzio di circa tre secoli è disceso sull'Olimpico. L'accademia dovette fare fronte a spese che misero in grave difficoltà le proprie finanze. D'altra parte l'edificazione del Teatro Olimpico aveva caratterizzato la fine di un florido periodo sperimentale, nel quale il teatro di ispirazione classica era stato riportato in vita; proprio come la rappresentazione inaugurale del teatro Olimpico, potrebbe essere considerata al contempo l'apice e la fine stessa di un periodo di sperimentazione durato due secoli. (Schiavo 1987: 51)

Che lo si voglia considerare uno spazio a cui si addica solo il silenzio (Barbieri 1974: 313), il monumento funebre più eloquente del Rinascimento (Flaiano 1967), un gran teatro della morte (Mazzoni 1998), o più ironicamente la materializzazione architettonica del vecchio servo di Don Rodrigo, sempre pronto ad essere "riesumato" per accogliere degnamente il notabile di turno, il Teatro Olimpico rimane al suo posto nonostante guerre, a cui è miracolosamente sopravvissuto, e restauri non sempre avveduti, forse perché:

"I teatri non furono mai costruiti per una sola epoca, ma per vivere nel tempo" (Poesio 1961).

9 G. Dolfin, Lettera del 9 marzo 1585, depositata presso la Biblioteca Bertoliana di Vicenza. 


\section{Ocula $\mathbf{a}^{13}$}

Architettura e politica: un incrocio di sguardi | Stefano Carlucci, II fiore di stucco

\section{Bibliografia}

AA.VV.

2003 Merriam-Webster'Collegiate Dictionary, Eleventh Edition.

AA. VV.

1967 The Oxford Companion to the theatre, edited by Phillis Hartnoll, O.U. P.

Ackermann, J.

1980 Palladio, trad. it. di G. Scattone, Torino, Einaudi, 1982.

Barba, E.

1993 La canoa di carta, Bologna, Il Mulino.

Barthes, R.

1982 "Droit dans les yeux" in Essais critiques III. L'obvie et l'obtus, Paris, Seuil, (tr. it. "Diritto negli occhi" in L'ovvio e l'ottuso, Torino, Einaudi, 1985).

Bernheimer, R.

1956 Theatrum Mundi, Art Bulletin, XXXVIII.

Beyer, A.

1984 Palladio. Il Teatro Olimpico Berlin, Wagenbach Klaus GmbH; tr.fr. Le

Théatre Olympique. Architecture triumphale pour une société Humaniste, Paris, Brio ed., 1989.

Buyssens, E.

1943 Les langages et le discours, Brussels, Office de Pubblicitè.

Carlson, $\mathrm{M}$.

1987 Places of Performance. The Semiotic of Theatre Architecture, Ithaca and London, Cornell University Press.

Eco, U.

1968 La struttura assente: introduzione alla ricerca semiologica, Milano, Bompiani.

Fils, R.

1777 Traité de la construction des théâtres et des machines théâtrales, Paris.

Hartnoll, P.

1967 The Concise History of Theatre, New York, Harry N. Abrams.

Ingegneri, A.

1598 Della poesia rappresentativa e del modo di rappresentare le favole sceniche, Modena, Panini, 1989.

Jacquot, J.

1964 Le lieu théâtral à la Renaissance, Paris, Éditions du Centre national de la recherche scientifique.

Kernodle, G.

1944 From Art to Theatre, Chicago, The University of Chicago Press.

Myers, H. G.

1978 Andrea Palladio: Activities with the Accademia Olimpica, Kent, Kent State University.

18 | www.ocula.it | agosto 2012 


\section{Ocula ${ }^{13}$}

Architettura e politica: un incrocio di sguardi | Stefano Carlucci, II fiore di stucco

Molinari, C.

1974 Bollettino del Centro Internazionale di Studi Andrea Palladio, Vicenza.

Palladio, A.

1570 Quattro libri dell'Architettura, Venezia, in Nalby D. Ewing, Andrea Palladio, 1508-1580: Architect and Humanist, Mosaic 7/4, summer 1974.

Patte, P.

1782 Essai sur l'architecture théâtrale, Paris.

Pavis, $\mathrm{P}$.

1989 Dictionnaire dù théatre, Paris, Edizioni Sociali Messidor.

Poesio, P. E.

1961 "Un uomo in ogni stagione”, La Nazione, 9 settembre.

Ponzio, A.

2004 Linguistica generale, scrittura letteraria e traduzione, Perugia, Guerra Edizioni.

Riccoboni, A.

1973 Lettera raccolta in, A. Gallo, La prima rappresentazione al Teatro Olimpico: con i progetti e le relazioni dei contemporanei, Milano, Il Polifilo.

Schiavo, R.

1987 Una guida al Teatro Olimpico, Vicenza, Accademia Olimpica di Vicenza Editrice.

Vitruvius

1960 The ten Books of Architecture, New York, Dover Publications, translated into English by M. H. Morgan.

Yates, F.

1969 Theatre of the World, London, Routledge \& Kegan.

Zorzi, G.

1965 Le opera pubbliche e $i$ palazzo di Andrea Palladio, Vicenza, Neri Pozza Editore. 